\title{
Correction \\ Correction: Nitrogen washout/washin, helium dilution and computed tomography in the assessment of end expiratory lung volume
}

\author{
Davide Chiumello ${ }^{1}$, Massimo Cressoni ${ }^{2}$, Monica Chierichetti ${ }^{2}$, Federica Tallarini ${ }^{2}$, Marco Botticelli², \\ Virna Berto ${ }^{2}$, Cristina Mietto ${ }^{2}$ and Luciano Gattinoni ${ }^{1,2}$

\begin{abstract}
1'Dipartimento di Anestesia, Rianimazione (Intensiva e Subintensiva) e Terapia del Dolore, Fondazione IRCCS - "Ospedale Maggiore Policlinico Mangiagalli Regina Elena", via Francesco Sforza 35, 20122, Milano, Italy

2Istituto di Anestesiologia e Rianimazione, Fondazione IRCCS - "Ospedale Maggiore Policlinico Mangiagalli Regina Elena" di Milano, Italy; Università degli Studi di Milano, via Festa del Perdono 7, 20122, Milano, Italy
\end{abstract}

Corresponding author: Luciano Gattinoni, gattinon@policlinico.mi.it

See related research by Chiumello et al., http://ccforum.com/content/12/6/R150

Published: 16 March 2009

Critical Care 2009, 13:405 (doi:10.1186/cc7743)

This article is online at http://ccforum.com/content/13/2/405

(c) 2009 BioMed Central Ltd

Following publication of our recent article [1], we noticed several errors relating to the figures. The complete set of correct figures follows below (Figure 1, 2, 3, 4 and 5).

The legend for Figure 5, 'Comparison of end expiratory lung volume (EELV) measured by the helium dilution technique and the nitrogen washout/washin method', was incorrect and should read as follows:

Comparison of end expiratory lung volume (EELV) measured by the helium dilution technique and the nitrogen washout/ washin method. (a) The EELV measured by the helium dilution as a function of the EELV measured by nitrogen washout/washin method (EELV helium dilution $=-111.85+$ $0.89 \times$ EELV GE, $r^{2}=0.82, p<0.00001$ ). (b) The BlandAltman plot of the EELV measured with the nitrogen washout/washin technique and the EELV measured with the helium dilution method. The $x$-axis shows the mean of the two measurements and the $y$-axis shows the difference between the EELV measured by then helium dilution method and the nitrogen washout/washin method (average difference -229 \pm $164 \mathrm{ml}$, limits of agreement $-558-100 \mathrm{ml}$ ).

The values in graph (a) of Figures 1, 4 and 5 had been plotted onto the incorrect axis.

The panels in Figure 3 had been switched. Graph (a) should be the linear regression plot and graph (b) should be the Bland-Altman plot.

\section{Reference}

1. Chiumello D, Cressoni M, Chierichetti M, Tallarini F, Botticelli M, Berto V, Mietto C, Gattinoni L: Nitrogen washout/washin, helium dilution and computed tomography in the assessment of end expiratory lung volume Critical Care 2008, 12:R150. 


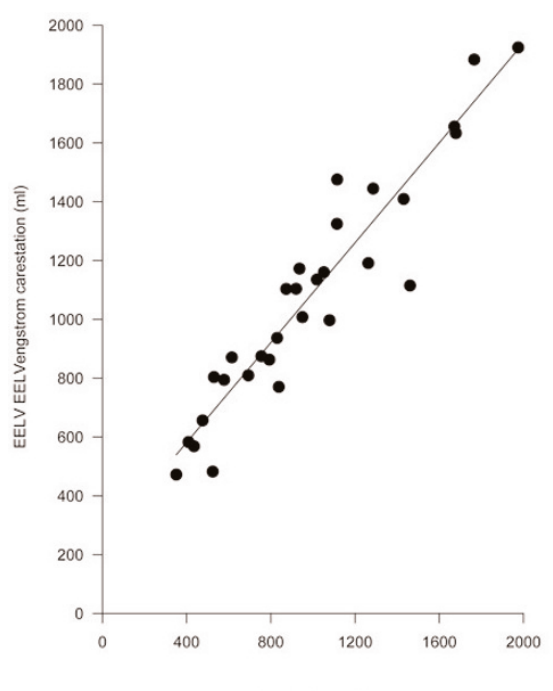

EELVCT scan (ml)

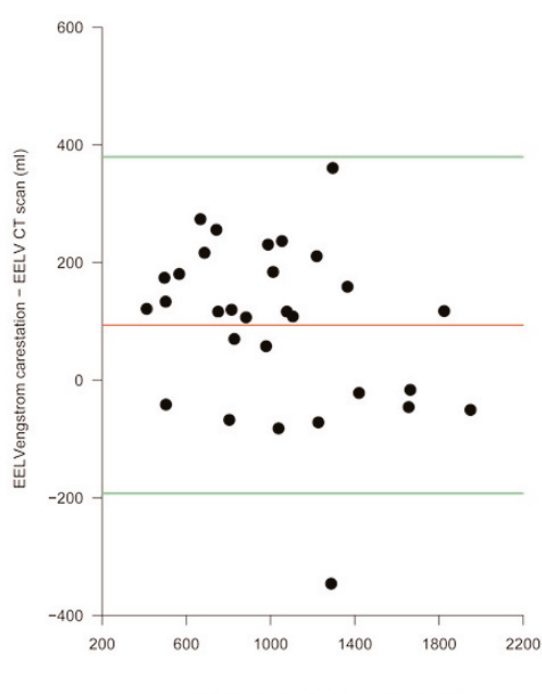

Mean of EELV Engstrom Carestation and EELV CT scan (ml)

Comparison of end expiratory lung volume (EELV) measured by the Engstrom Carestation and the computed tomography (CT) scan. (a)The EELV measured by the Engstrom Carestation as a function of the EELV measured by the computed tomography (CT) scan (EELV carestation $=242+0.85 \times$ EELV CT scan, $r^{2}=0.89, p<0.00001$ ). (b) The Bland-Altman plot of the EELV measured with the CT scan and the EELV measured with the Engstrom Carestation. The $x$ axis shows the mean of the two measurement and the $y$ axis shows the difference between the EELV measured by the Engstrom Carestation and the EELV measured by the CT scan (average difference $93 \pm 143 \mathrm{ml}$, limits of agreement $-50-236 \mathrm{ml}$ ).

\section{Figure 2}

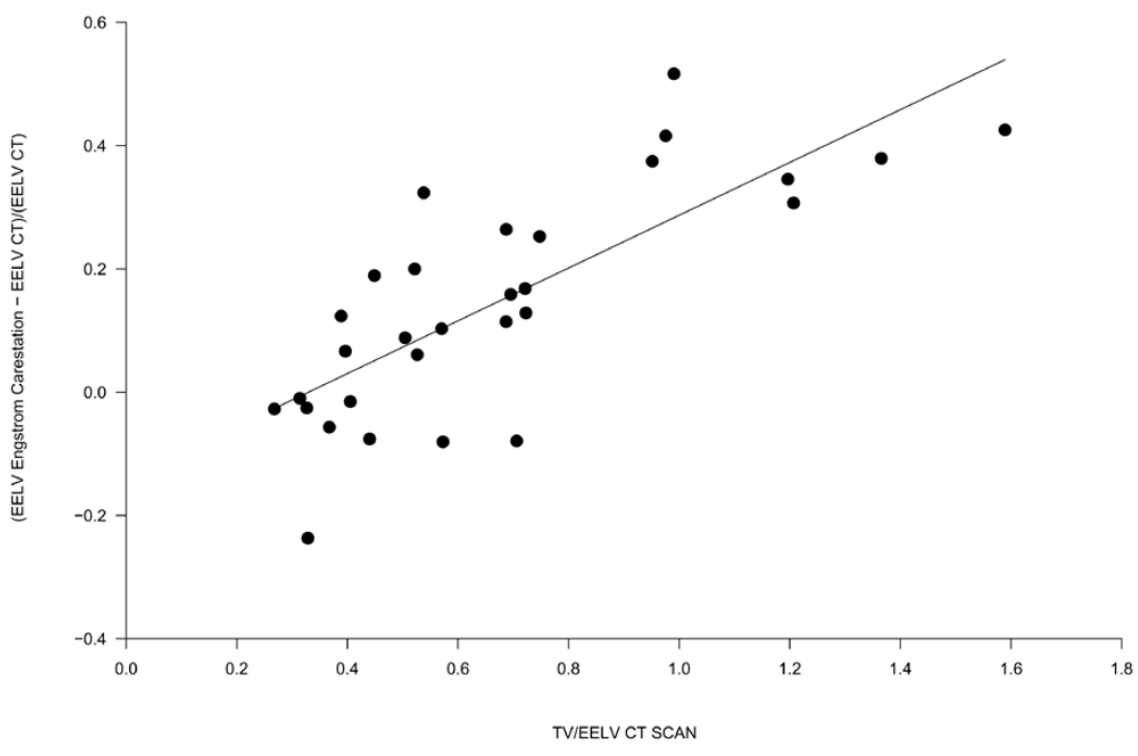

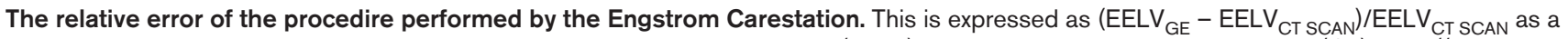
function of the ratio between tidal volume and the end expiratory lung volume (EELV) measured by computed tomography (CT) scan ((EELV $\mathrm{GE}-$ $\left.\mathrm{EELV}_{\text {CT SCAN }}\right) / \mathrm{EELV}_{\text {CT SCAN }}=0.05+0.43 \times($ Tidal Volume/EELV 

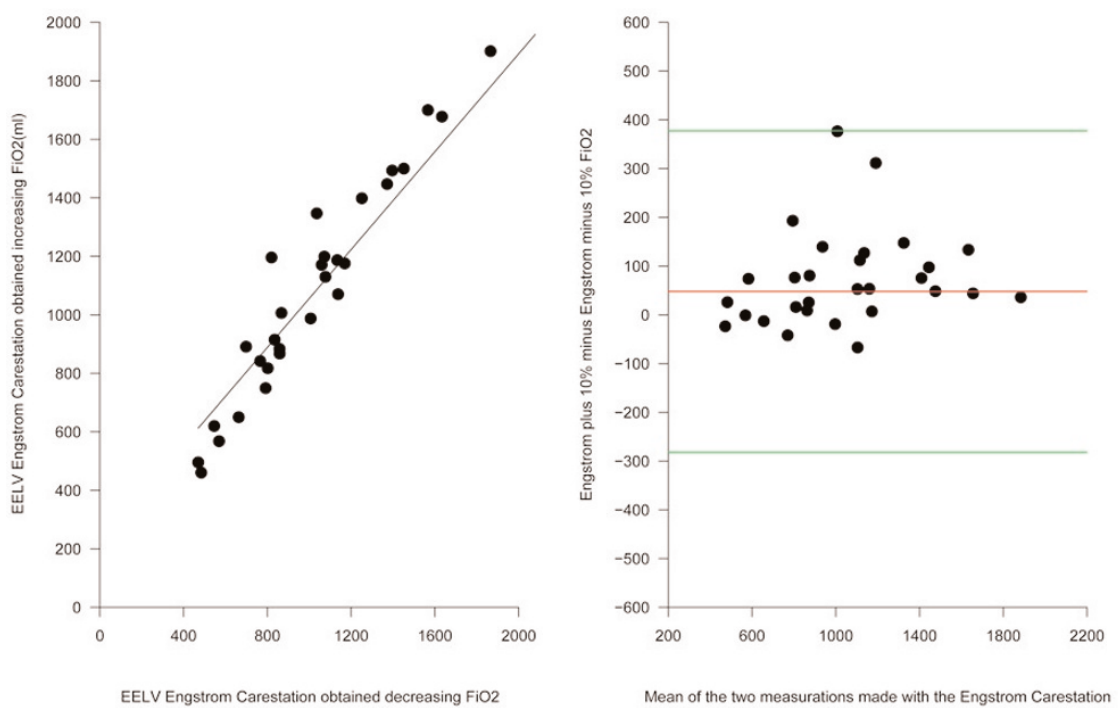

Accuracy of the nitrogen washin-washout technique. (a) The relation between the EELV measured by increasing the $\mathrm{FiO}_{2}$ as a function of the EELV obtained decreasing $\mathrm{FiO}_{2}$. The EELV obtained increasing the $\mathrm{FiO}_{2}$ was $-56+1.0078$ multiplied by the EELV obtained decreasing the $\mathrm{FiO}{ }_{2}$ $\left(r^{2}=0.84, p<0.0001\right)$. (b) The Bland-Altman plot of the EELV measurement obtained increasing the $\mathrm{FiO}_{2}$ and the EELV obtained decreasing the $\mathrm{FiO}_{2}$. The $\mathrm{x}$ axis shows the mean of the two measurements and the difference between the EELV measured by increasing $\mathrm{FiO}_{2}$ and the $y$ axis shows the EELV obtained decreasing $\mathrm{FiO}_{2}$ (average difference $48 \pm 165 \mathrm{ml}$, limits of agreement -117-213 ml).

\section{Figure 4}

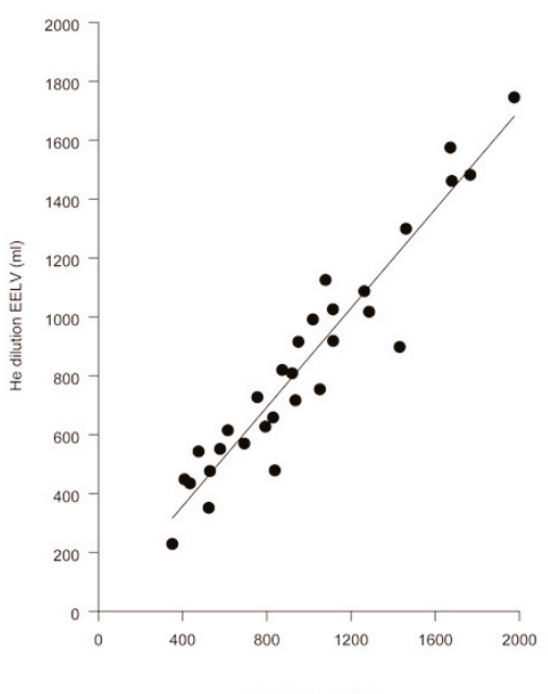

EELVCT scan (ml)

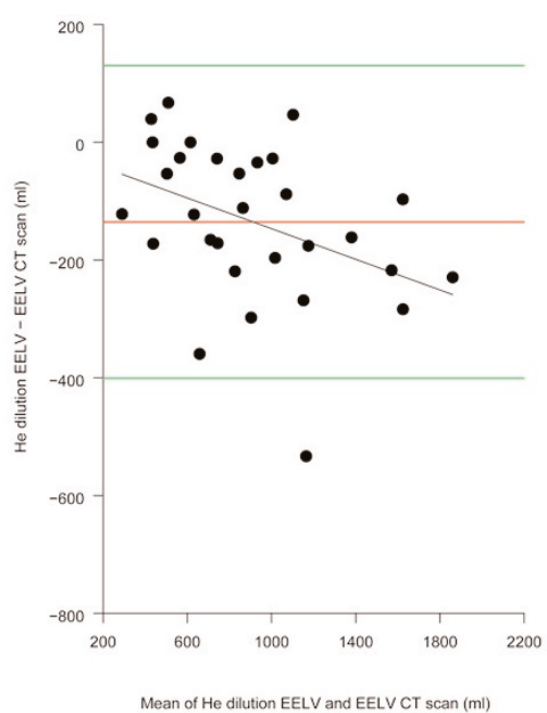

Comparison of end expiratory lung volume (EELV) measured by the helium dilution technique and the computed tomography (CT) scan. (a) The EELV measured by the helium dilution technique as a function of the EELV measured by the CT scan (EELV helium dilution $=20+0.84 \times$ EELV CT scan, $r^{2}=0.91, p<0.00001$ ). (b) The Bland-Altman plot of the EELV measured with the CT scan and the EELV measured with the helium dilution method. The $x$ axis shows the mean of the two measurements and the $y$ axis shows the difference between the EELV measured by the helium dilution method and the EELV measured by the CT scan (average difference $-136 \pm 133 \mathrm{ml}$, limits of agreement $-3-269 \mathrm{ml}$ ). The difference between the EELV measured with the helium dilution method and the EELV measured with CT scan was significantly correlated with the EELV, expressed as the average between the two measurements (Helium EELV - CT scan EELV $=-15.52764+-0.17034 \times($ helium EELV + CT scan EELV) $/ 2, r^{2}=0.21, p=0.005838$ ). 
Figure 5
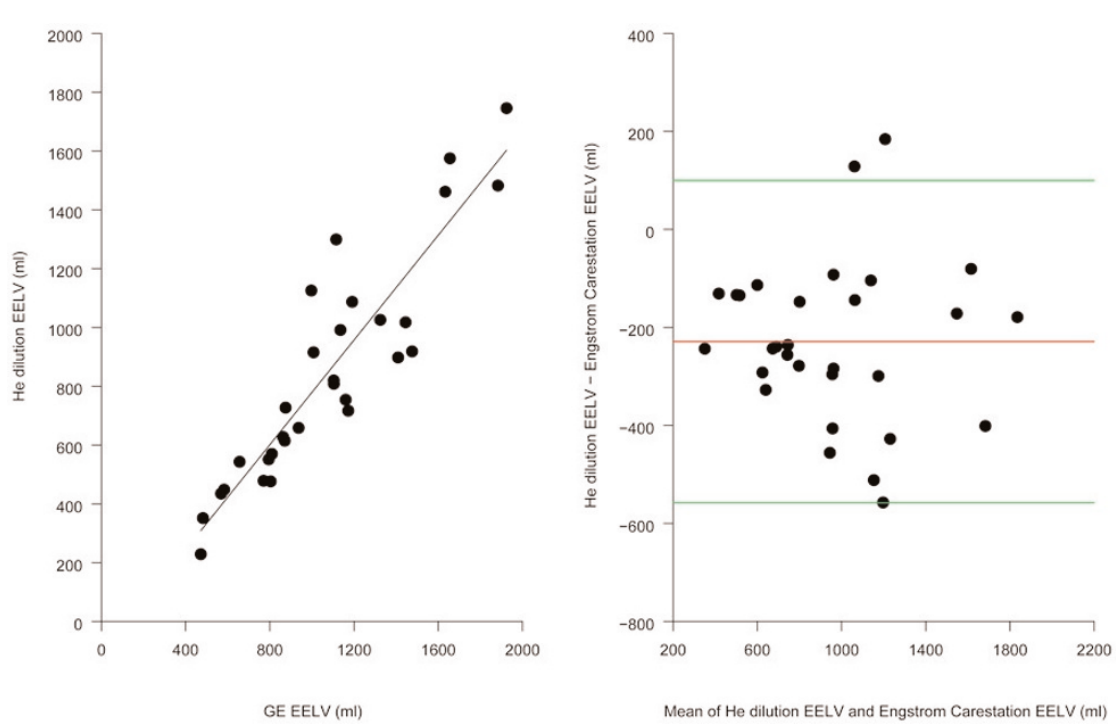

Comparison of end expiratory lung volume (EELV) measured by the helium dilution technique and the nitrogen washout/washin method. (a) The EELV measured by the helium dilution as a function of the EELV measured by nitrogen washout/washin method (EELV helium dilution = $-111.85+0.89 \times$ EELV GE, $r^{2}=0.82, p<0.00001$ ). (b) The Bland-Altman plot of the EELV measured with the nitrogen washout/washin technique and the EELV measured with the helium dilution method. The $x$-axis shows the mean of the two measurements and the $y$-axis shows the difference between the EELV measured by then helium dilution method and the nitrogen washout/washin method (average difference $-229 \pm 164$ $\mathrm{ml}$, limits of agreement $-558-100 \mathrm{ml})$. 